\title{
Die Auferweckung des Lazarus in den gottesdienstlichen Hymnen der Orthodoxen Kirche
}

Ein Beitrag zur Auslegungsgeschichte von Joh 11,1-44

\author{
OTFried Hofius*
}

This essay investigates the liturgical hymns of the Orthodox Church referring to the Johannine story of the Raising of Lazarus (Jn. 11:1-44). These hymns are especially sung at the Services of the Saturday before Palm Sunday known as the Saturday of the holy and righteous Lazarus. The relevant hymns are deep theological interpretations of the Johannine story and therefore an important contribution to the history of exegesis of the Gospel according to St John.

Keywords: Gospel according to St John, The Raising of Lazarus, Orthodox Church, Orthodox liturgical hymns, History of exegesis of the Fourth Gospel

Die Erzählung von der Auferweckung des Lazarus Joh 11,1-44 gehört sowohl in narrativer wie auch in theologischer Hinsicht zu den zentralen Texten des Vierten Evangeliums und des Neuen Testaments überhaupt. Die Orthodoxe Kirche hat - in auffallendem Unterschied zu den Kirchen des Westens - das Zeugnis dieser Erzählung dadurch in eindrucksvoller Weise aufgenommen, dass dem in ihr berichteten Geschehen innerhalb des Kirchenjahrs ein eigener Festtag gewidmet worden ist: der „Samstag des heiligen und gerechten Lazarus"1. Dieser Tag, an dem die Erzählung von der Auferweckung des Lazarus in der Göttlichen Liturgie vollständig verlesen wird, gehört zu den hohen Festen der Kirche². An ihm - aber auch in den Gottesdiensten der fünf voraufgehenden Werktage und des unmittelbar folgenden Palmsonntags - werden darüber hinaus zahlreiche Hym-

* Otfried Hofius, Prof. Dr. theol., Evangelisch-theologische Fakultät der Universität Tübingen. Privatadresse: Im Kleinen Feldle 11, D-72072 Tübingen; e-mail: o.hofius@t-online.de.

1 Siehe dazu den entsprechenden Artikel in: Konrad Onasch, Kunst und Liturgie der Ostkirche in Stichworten unter Berücksichtigung der Alten Kirche, Wien - Köln - Graz 1981, S. $238 f$.

2 In der älteren Tradition wird der Lazarussamstag in der Regel zum Dodekaortion gerechnet; siehe: K. Onasch, „Dodekaortion“, in: idem, Kunst und Liturgie der Ostkirche, S. 86. Zur heute üblichen Bestimmung der zwölf Hochfeste siehe etwa: The Festal Menaion, translated from the original Greek by Mother Mary and Archimandrite Kallistos Ware, London Boston 1977/1984, S. 41f.; Peter D. Day, „Twelve great feasts“, in: idem, The Liturgical Dictionary of Eastern Christianity, Collegeville, Minnesota 1993, S. 296. 
nen gesungen, die das Wunder der Auferweckung des Lazarus zum Thema haben ${ }^{3}$. Diesen Hymnen, in denen wir höchst beachtliche Dokumente der Auslegungs- und Wirkungsgeschichte der johanneischen Erzählung zu erkennen haben ${ }^{4}$, sind die folgenden Darlegungen gewidmet ${ }^{5}$. Unser Interesse gilt dabei der Frage, welche Züge und Aussagen des Evangelienberichts in den Hymnen aufgenommen wurden, welche Deutung sie dort erfahren haben und wie diese Deutung im Licht heutiger wissenschaftlicher Exegese des Textes Joh 11,1-44 zu würdigen ist ${ }^{6}$.

Der Betrachtung der Hymnen sei eine knappe Übersicht über die relevanten Texte vorangestellt ${ }^{7}$. Den größten Teil des Materials liefern die folgen-

3 Neben den Lazarus-Hymnen, die in das Triodion - das liturgische Buch mit den Eigentexten für die Vorfastenzeit und die Große Fastenzeit - aufgenommenen wurden, sind weitere Dichtungen erhalten. Erwähnt seien die beiden Hymnen des Romanos Melodos (6. Jh.): Paul Maas, Constantine A. Trypanis (Hrsg.), Sancta Romani Melodi Cantica. Cantica genuina, Oxford 1963, S. 102-116; José Grosdidier de Matons (Hrsg.), Romanos le Mélode, Hymnes III (SC 114), Paris 1965, S. 145-225, 154-179, 198-219.

4 Zur Auslegungs- und Wirkungsgeschichte von Joh 11,1-44 siehe: Jacob Kremer, Lazarus. Die Geschichte einer Auferstehung. Text, Wirkungsgeschichte und Botschaft von Joh 11,1-46, Stuttgart 1985. Kremer weist dort lediglich auf Romanos Melodos hin (S. 150-152); die in dem vorliegenden Aufsatz behandelte Hymnendichtung findet dagegen keine Berücksichtigung.

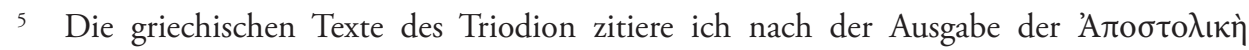

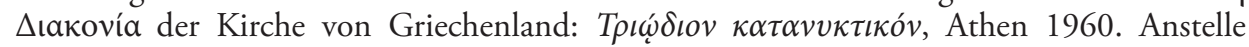
der dort gebotenen metrischen Abteilung der einzelnen Verszeilen wähle ich jedoch eine der syntaktischen Struktur der Sätze entsprechende Interpunktion. Allen griechischen Hymnen-Zitaten habe ich eine deutsche Übersetzung beigefügt, die um eine möglichst genaue Wiedergabe des Textsinns bemüht ist. Folgende Übersetzungen wurden verglichen: Die Ostkirche betet. Hymnen aus den Tagzeiten der Byzantinischen Kirche II: Vierte bis sechste Fastenwoche. Die Heilige Woche, Münster ${ }^{2} 1963$ (Übersetzung von Kilian Kirchhoff, Überarbeitung von Chrysologus Schollmeyer); Der Gottesdienst am Samstag des hl. gerechten Lazarus. Zusammengestellt und übersetzt von Erzpriester Dimitrij Ignatiev, München 1991; The Lenten Triodion, translated from the original Greek by Mother Mary and Archimandrite Kallistos Ware, London - Boston 1978, South Canaan, PA 2002; The Lenten Triodion. Supplementary Texts, translated from the original Greek by Mother Mary and Archimandrite Kallistos Ware, Bussy-en-Othe 1979.

6 Zur Exegese siehe: Otfried Hofius, „Die Auferweckung des Lazarus. Joh 11,1-44 als Zeugnis narrativer Christologie“, in: idem, Exegetische Studien (WUNT 223), Tübingen 2008, S. 28 45. In diesem Aufsatz finden die im Folgenden notierten exegetischen Feststellungen ihre detaillierte Begründung. Vergleiche außerdem auch die tiefgreifenden Ausführungen zu Joh

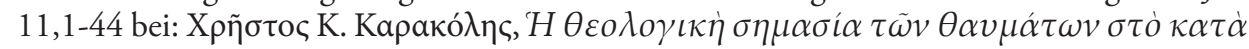

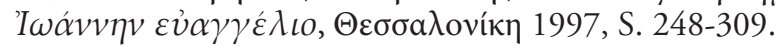

7 Die Übersicht berücksichtigt, dass in liturgischer Hinsicht die Gottesdienste des Freitagabends zum Lazarussamstag und diejenigen des Samstagabends zum Palmsonntag

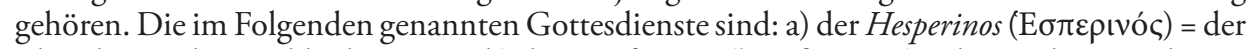
Abendgottesdienst, d.h. die Vesper; b) das Apodeipnon ('A dem in der westlichen Tradition die Komplet entspricht; c) der Orthros ('O $\rho \theta \rho \circ \varsigma)=$ der Matutin 
den Dichtungen, die jeweils einem bedeutenden Hymnographen zugeschrieben werden ${ }^{8}$ :

\section{Hymnen des Lazarussamstags:}

- im Hesperinos: Stichira Kaiser Leons des Weisen

- im Apodeipnon: Kanon des Andreas von Kreta

- im Orthros: $\quad$ Kanon des Theophanes Graptos

Kanon und Tetraodion des Kosmas von Majuma?

Tetraodion und Stichira des Johannes Monachos ${ }^{10}$

Hymnen der fünf dem Fest voraufgehenden Tage ${ }^{11}$ :

- im Orthros: $\quad$ Triodia des Joseph von Thessaloniki

und des Theodoros Studites

und Laudes entsprechende Morgengottesdienst. Während ich bei den drei Gottesdiensten sowie bei den Namen der Hymnographen der erasmischen Aussprache des Griechischen folge, werden andere griechische Fachbegriffe - von den Termini „Ode“ und „Stichologie“ abgesehen - nach der neugriechischen Aussprache wiedergegeben. Eine knappe Erläuterung der hymnologischen bzw. liturgischen Begriffe bietet der terminologische Anhang.

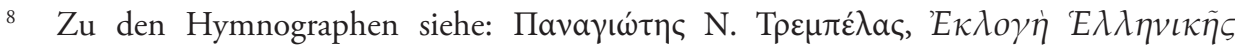

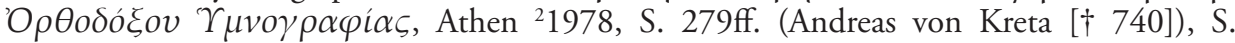
310ff. (Kosmas von Majuma [8. Jh.]), S. 321f. (Theophanes Graptos [† 845]), S. $345 f$. (Theodoros Studites [759-826] und Joseph von Thessaloniki [762-832]), S. 376 (Leon der Weise [886-912]); siehe auch S. 287ff. zu Johannes Damaskenos ( $†$ vor 754) und zur Frage der Identifizierung des Johannes Monachos. Nicht näher bekannt ist offensichtlich Andreas der Blinde (Andreas Typhlos), dessen Stichiron in Fußnote 19 erwähnt wird. Knappe Informationen zu den Hymnographen finden sich in: Kilian Kirchhoff, Chrysologus Schollmeyer, Die Ostkirche betet. Hymnen aus den Tagzeiten der Byzantinischen Kirche I: Vorfastenzeit. Erste bis dritte Fastenwoche, Münster ${ }^{2}$ 1962, S. 451-453; K. Kirchhoff, Osterjubel der Ostkirche. Hymnen aus der fünfzigtägigen Osterfeier der Byzantinischen Kirche, Münster ${ }^{2}$ 1961, S. 621-623; K. Kirchhoff, Ch. Schollmeyer, Hymnen der Ostkirche. Dreifaltigkeits-, Marien- und Totenhymnen, Münster ${ }^{2} 1960 / 1979$, S. 269 f.

9 Von Kosmas stammt auch der im Orthros des Palmsonntags gesungene Kanon, in dessen dritter Ode von Lazarus die Rede ist.

10 Die in Fußnote 5 genannte Edition des Triodion bietet bei den mit den Äni verbundenen Stichira keine Verfasserangabe. Dagegen wird Johannes Monachos als Autor genannt in:

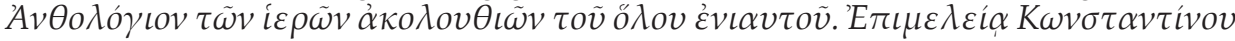

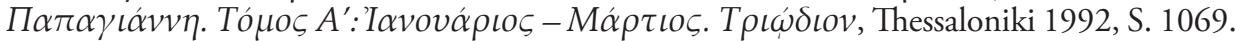

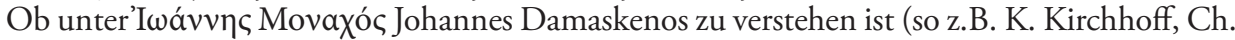
Schollmeyer, Die Ostkirche betet II, S. 323-327; K. Onasch, Kunst und Liturgie, S. 239), ist

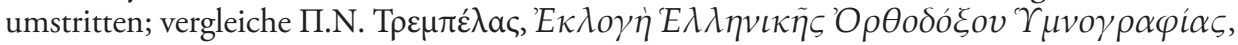
S. 287ff. Ich führe deshalb bei der Verfasserangabe lediglich den Namen Johannes an.

11 Bei den im Orthros auf die Stichologien folgenden Kathismen fehlt in der in Fußnote 5 genannten Edition des Triodion eine Verfasserangabe, wohingegen diese in Die Ostkirche betet II (siehe ebd.) Joseph von Thessaloniki bzw. Theodoros Studites zugeschrieben werden. - Da der Hesperinos des Freitags bereits zum Lazarussamstag zählt, gilt das zu diesem Gottesdienst Gesagte nur für die Tage von Montag bis Donnerstag. 


\section{- im Hesperinos: Stichira des Joseph von Thessaloniki und des Theodoros Studites.}

Diesen Dichtungen treten die anonym überlieferten Hymnen an die Seite, zu denen unter anderem das Festtroparion und das Kontakion des Lazarussamstags sowie bestimmte Kathismata und Stichira des Palmsonntags gehören ${ }^{12}$.

\section{II}

Wenn wir uns nunmehr den Texten selbst zuwenden, so ist in einem ersten Schritt unserer Betrachtung auf drei erzählerische Motive hinzuweisen, denen im Gesamtzusammenhang von Joh 11,1-44 eine besondere Bedeutung zukommt und die in den Hymnen sowohl aufgenommen wie auch in ihrer theologischen Bedeutung reflektiert werden ${ }^{13}$.

1. Die Hymnen bringen das wichtige Motiv zur Sprache, das in dem Abschnitt Joh 11,6-16 zum Ausdruck kommt. Wie der Evangelist in diesen Versen darlegt, ist der Weg zur Auferweckung des Lazarus für Jesus selbst der Weg, der ihn an das Kreuz führen wird ${ }^{14}$. Das aber bedeutet: Damit Lazarus lebt, muss Jesus sterben. Diesen Zusammenhang beschreibt unter anderem ein Triodion des Joseph. Der Dichter erwähnt zunächst die Ankündigung Jesu, dass er nach Jerusalem gehe, um am Kreuz zu sterben ${ }^{15}$, und sodann heißt es:

12 Was die Fundorte der im Folgenden zitierten bzw. erwähnten Texte anlangt, so gilt: a) Die Hymnen des Lazarussamstags werden stets an erster Stelle sowie ohne Erwähnung des Tages und des entsprechenden Gottesdienstes angeführt. b) Bei den Hymnen der fünf dem Lazarussamstag vorausgehenden Werktage und des Palmsonntags werden jeweils der Tag und der betreffende Gottesdienst genannt. c) Oden werden mit römischen, Strophen (Troparia) mit arabischen Zahlen bezeichnet. Die Strophenzählung entspricht der im Triodion (siehe Fußnote 5) gebotenen Abfolge; dabei wird der Irmos, die Leitstrophe, stets als erste Strophe gezählt - also auch dann, wenn dort nur sein Anfang angegeben ist.

13 Daneben wären weitere Motive zu notieren, die aber von geringerem Gewicht sind und deshalb in dem vorliegenden Aufsatz nicht erörtert werden sollen. Nur ein Beispiel sei genannt: In Aufnahme der Wendung $\Lambda a ́ \zeta \alpha \rho o \varsigma ~ o ́ ~ \varphi i ́ \lambda o \varsigma ~ i j \mu \tilde{\omega} v$ Joh 11,11 wird Lazarus in den Hymnen mehrfach als Jesu „Freund“ bezeichnet, und die entsprechende Aussage, dass Jesus Lazarus „lieb hatte“ (Joh 11,3b.5), findet etwa ihr Echo im Tetraodion des Kosmas

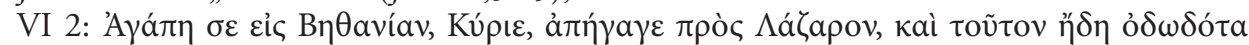

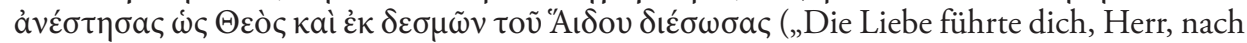
Bethanien zu Lazarus, und ihn, der schon verweste, hast als Gott du auferweckt und aus den Fesseln des Hades errettet").

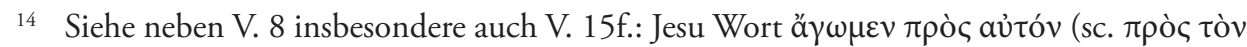

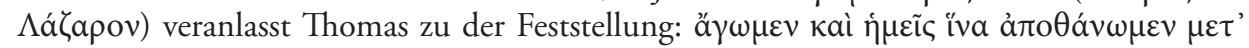
aủนoũ.

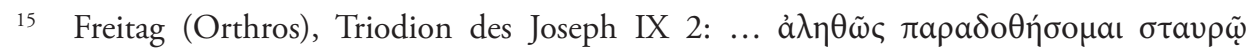

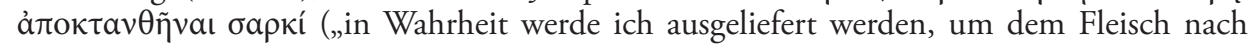
am Kreuz getötet zu werden“). Hier handelt es sich um eine Bezugnahme auf Mt 20,18f. (vergleiche Mk 10,33f.; Lk 18,31-33). 


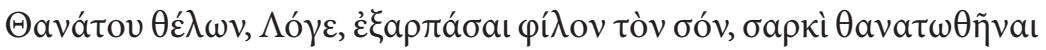

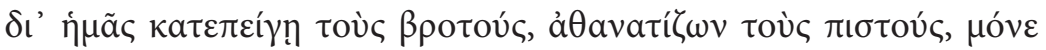

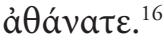

„Weil du, o Wort, deinen Freund dem Tod entreißen willst, begehrst $\mathrm{du}$, dich um unsertwillen, die wir sterblich sind, dem Fleisch nach töten zu lassen und so die Glaubenden unsterblich zu machen, du allein Unsterblicher."

Als höchst bedeutsam muss gelten, dass das Troparion nicht nur von Lazarus spricht, sondern zugleich auch von „uns“, d.h. von den an Christus Glaubenden. Das entspricht voll und ganz dem Sinn der Erzählung Joh 11,1-44 selbst. In ihr geht es keineswegs nur um Lazarus allein; Lazarus repräsentiert vielmehr alle, die dem Tod verfallen sind und für die Christus in freiwilliger Entscheidung den Weg an das Kreuz geht, um sie aus der Gewalt des Todes zu erretten. Dieser Gedanke begegnet uns auch in dem folgenden Troparion, das ausdrücklich auf Joh 11,8 Bezug nimmt:

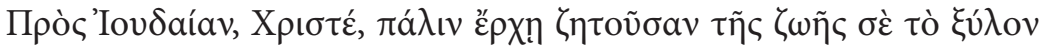

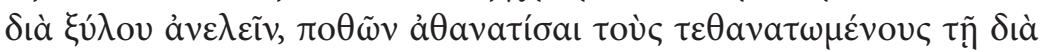
$\xi \hat{\lambda} \lambda$ ov $\beta \rho \omega ́ \sigma \varepsilon$ I. $^{17}$

„Nach Judäa, das dich, den Baum des Lebens, durch den Baum (sc. des Kreuzes) töten will, gehst du aufs Neue, Christus, weil du die unsterblich machen willst, die durch die Speise des Baumes getötet worden sind."

Ein anderes Troparion kann dementsprechend dem einzelnen Gläubigen die Aussage in den Mund legen, dass Jesus nach Jerusalem geht,

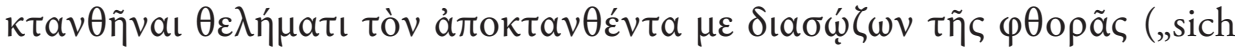
freiwillig töten zu lassen, um mich, den Getöteten, vom Verderben zu erretten") ${ }^{18}$.

2. Die Hymnen betonen in Aufnahme der erzählerischen Notiz von Joh 11,43f., dass die Auferweckung des Lazarus durch Jesu „Wort“, „Stimme“ oder

\footnotetext{
16 Freitag (Orthros), Triodion des Joseph IX 3.

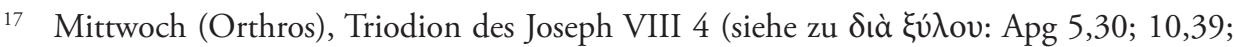

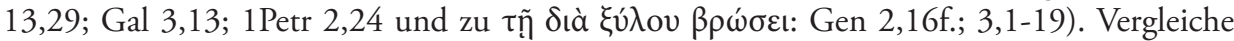
auch Mittwoch (Orthros), Triodion des Theodoros IX 3: Nach Judäa, das ihn zu steinigen

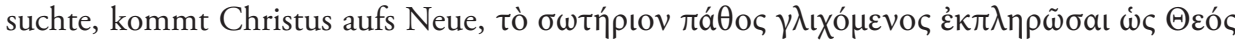
(,in dem Verlangen, das erlösende Leiden als Gott zu vollenden“), und er erduldet dort willig

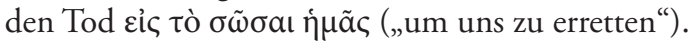

18 Freitag (Orthros), Triodion des Joseph V 3. Siehe ferner noch: Stichira des Leon 1; Tetraodion des Johannes VII 2; Mittwoch (Orthros), Triodion des Joseph VIII 4.
} 
„Befehl“"vollzogen wird ${ }^{19}$. Nähere Kennzeichnungen sind dabei die in Anreden

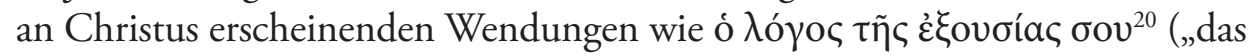

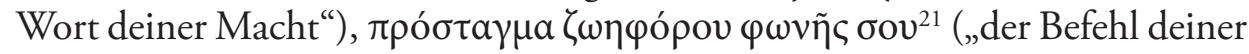

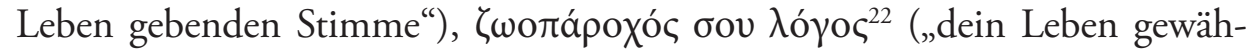

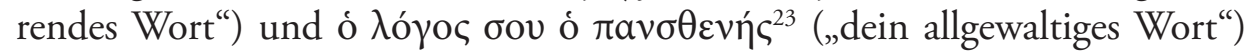

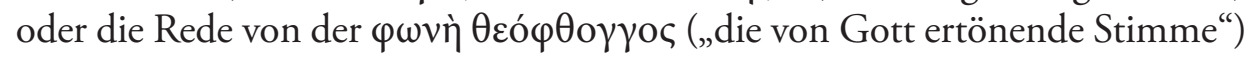
und dem $\theta \varepsilon \ddot{k}$ òv $\dot{\rho} \tilde{\mu} \alpha$ („das göttliche Wort“) Christi ${ }^{24}$. Insbesondere die Aus-

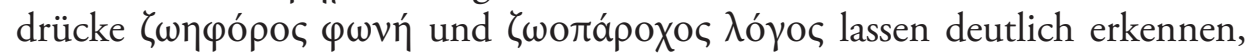
dass die Hymnendichter das Jesuswort Joh 5,25 mit im Blick haben, an das auch die Lazarus-Erzählung selbst unüberhörbar erinnert und erinnern will. In der Erzählung wird ja genau jener Sachverhalt beschrieben, der in dem genannten Wort Jesu in einer theologischen Aussage zur Sprache kommt: ả $\mu \grave{\eta} v$

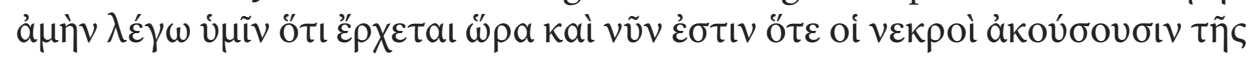

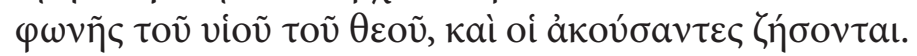

3. Die Hymnen bringen die Größe des Wunders der Auferweckung des Lazarus zum Ausdruck, indem sie im Anschluss an Joh 11,39 und Joh 11,43f. zwei erzählerische Motive aufnehmen, die beide in dem folgenden Troparion erwähnt werden:

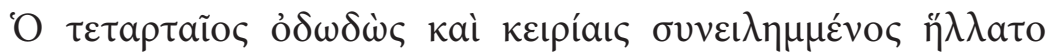

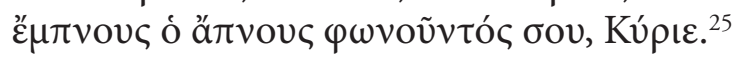

„Der bereits vier Tage Tote, der schon verweste und von Binden umwunden war, - er, der Entseelte, sprang beseelt umher, als du ihn riefst, o Herr."

Das eine Motiv betrifft den Tatbestand, dass Lazarus schon den vierten Tag im Grab liegt und also bereits in Verwesung begriffen ist. Immer wieder er-

19 Stichira des Leon 3.6; Stichiron Andreas des Blinden; Kanon des Andreas II 2-4.6-9. III 3-7. IV 3.4.10. V 3-5. VI 2-6. VII 8. VIII 3. IX 8; Kathismata nach der ersten und zweiten Stichologie; Kanon des Theophanes I 3. III 5. IV 4. V 2; Kanon des Kosmas III 4. IV 5. V 2.3; Anderes Kathisma nach Ode III; Tetraodion des Kosmas VI 3. VII 3. VIII 3; Tetraodion des Johannes VII 3. VIII 2; Exapostilarion; Stichira des Johannes 7-9; Palmsonntag (Großer Hesperinos), Stichira zu den Luzernariumspsalmen 4; Palmsonntag (Orthros), Kanon des

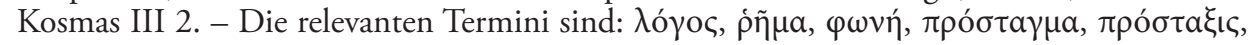

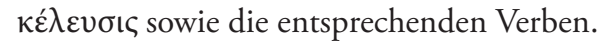

20 Stichira des Leon 3.

21 Kanon des Andreas II 4.

22 Mittwoch (Orthros), Triodion des Joseph IX 3.

23 Kanon des Kosmas III 4.

24 Kanon des Kosmas V 3 bzw. Kanon des Theophanes III 5.

25 Kanon des Andreas VIII 3; vergleiche Tetraodion des Johannes VII 4. 
scheint in den Hymnen das $\tau \varepsilon \tau \alpha \rho \tau$ aĩo von Joh 11,39b, und mehrfach begegnet daneben - wie in dem soeben zitierten Troparion - das Partizip ỏ $\delta \omega \delta \omega \varsigma^{26}$,

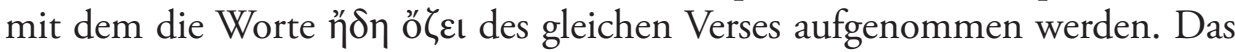
andere Motiv liegt in dem, was in Joh 11,43f. über den gebietenden Ruf Jesu

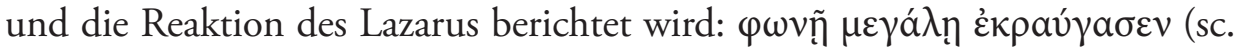

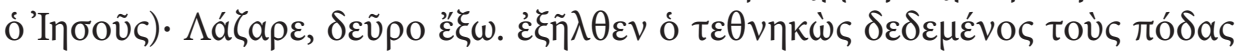

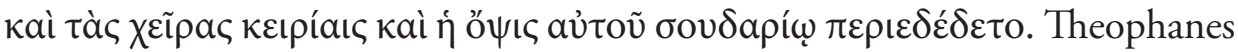
sagt dazu in einem seiner Troparia:

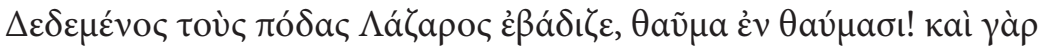

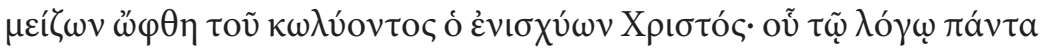

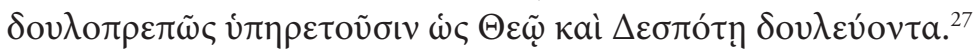

„Gebunden an den Füßen schritt Lazarus einher - Wunder aller Wunder! Denn als größer als der, der ihn zurückhalten wollte, zeigte sich der, der ihm Kraft verlieh: Christus. Seinem Wort gehorchen, wie es sich einem Sklaven geziemt, alle Dinge, und sie dienen ihm als Gott und Herrn."

In diesem Troparion ist nicht von einem zusätzlichen Wunder - also ei-

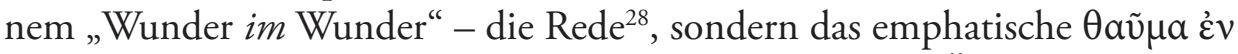

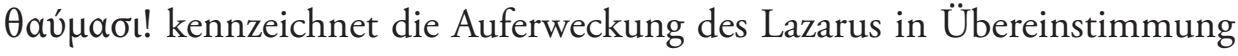
mit dem Sinn der Schilderung von Joh 11,44 als ein einzigartiges und unerhörtes Wunder, als das Wunder schlechthin ${ }^{29}$.

\section{III}

Bleiben wir bei den erzählerischen Motiven, so muss jetzt von jenen Hymnen gesprochen werden, die in dem Bericht von der Auferweckung des Lazarus ein Zeugnis dafür erkennen, dass Jesus im Sinne des Dogmas von Chalcedon beides ist: wahrer Gott und wahrer Mensch. Er selbst offenbart

26 Kanon des Andreas II 4.7. IV 7. VIII 3; Kanon des Kosmas I 4; Kanon des Theophanes V 4; Tetraodion des Kosmas VI 2; Tetraodion des Johannes VII 4; Palmsonntag (Großer Hesperinos), Stichira zu den Luzernariumspsalmen 4. - Die genaue Bedeutung von ỏ $\delta \omega \delta \omega \varsigma$ ist: „stinkend“; ich wähle dafür die Übersetzung „verwesend“ u.ä.

27 Kanon des Theophanes V 3. Zu dem Motiv von Joh 11,44 siehe ferner: Kanon des Andreas V 5; Tetraodion des Kosmas VII 3; Tetraodion des Johannes VII 4.

28 Diese Deutung findet sich bei einigen Kirchenvätern; siehe dazu: Walter Bauer, Das

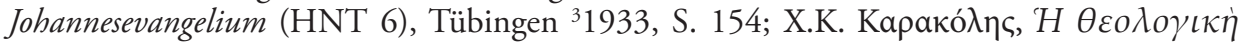

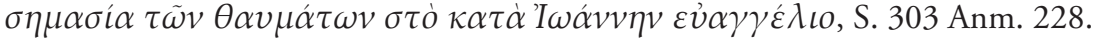

29 So richtig die Übersetzung in The Lenten Triodion, S. 481: „O wonder of wonders!“ Der

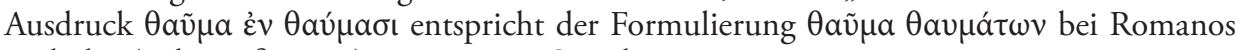
Melodos (siehe Fußnote 3), Hymnus II, Strophe 1. 
diesen Hymnen zufolge in dem in Joh 11,1-44 berichteten Geschehen sowohl seine wahre Gottheit wie auch seine wahre Menschheit. So heißt es in einem Troparion des Theophanes:

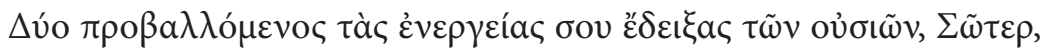

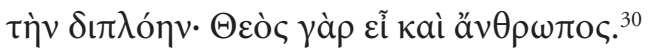

„Indem du deine zwei Tätigkeiten in Erscheinung treten ließest, hast du, Heiland, die Zweiheit deiner Wesenheiten zu erkennen gegeben; denn du bist Gott und Mensch."

Seine wahre Gottheit offenbart Jesus den Hymnen zufolge bereits darin, dass er von dem Tod des Lazarus weiß und ihn seinen Jüngern kundtut (Joh 11,11.14) ${ }^{31}$. Vor allem aber stellt er "die unbegrenzte Wirkkraft“ seiner Gottheit unter Beweis ${ }^{32}$, indem er „in eigener Machtvollkommenheit “33 den von der Verwesung gezeichneten Lazarus durch sein allgewaltiges Schöpferwort ${ }^{34}$ vom Tode auferweckt ${ }^{35}$. Darin zeigt sich, dass er der Sohn Gottes ${ }^{36}$ und als solcher - wie sein Vater - der „Gott der Lebenden und der Toten “ ist ${ }^{37}$. In der zuletzt erwähnten Aussage haben wir eine Prädikation vor uns, die Jesu einzigartige göttliche Hoheit zum Ausdruck bringt. Ihr treten in den Hymnen weitere Hoheitsbegriffe an die Seite. Neben dem häufigen $\Sigma \omega \tau$ ŕ $\rho$ und dem in Joh 1,1.14 vorgegebenen $\Lambda$ ó $\gamma o \varsigma^{38}$ sind etwa

30 Kanon des Theophanes III 2; siehe ferner: Kanon des Andreas IV 9. IX 3; Stichira des

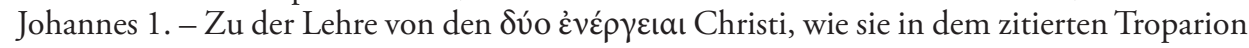
des Theophanes zum Ausdruck kommt, siehe das Glaubensbekenntnis (Versio graeca) und die Canones 11-16 des Concilium Lateranense von 649: DH 500. 511-516.

31 Kanon des Andreas I 8; Kanon des Kosmas I 2; Anderes Kathisma nach Ode III; Tetraodion des Johannes VI 2; Ikos; Stichira des Johannes 4; Mittwoch (Hesperinos), Stichira des Joseph 1.

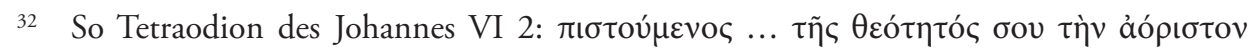

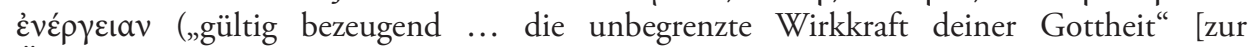

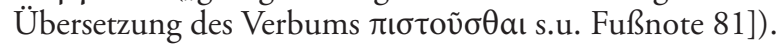

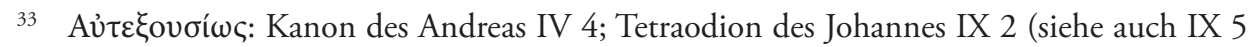

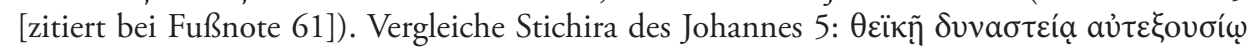

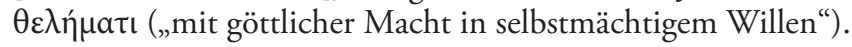

34 Siehe dazu die in den Fußnoten 19-24 notierten Belege.

35 Stichira des Leon 5; Kanon des Andreas III 5. IV 5.6.9. VII 8. IX 3; Kanon des Theophanes I 4; Tetraodion des Kosmas VI 2.3. VII 2. VIII 3; Tetraodion des Johannes VI 3. VIII 2.4. IX 4; Stichira des Johannes 1; Dienstag (Orthros), Triodion des Theodoros IX 3.

36 Kanon des Andreas IV 2.

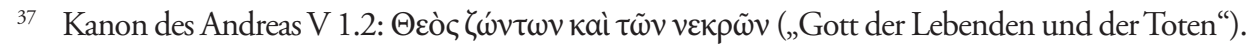

38 Kanon des Andreas IV 9. V 4. IX 3.8; Kanon des Kosmas I 4; Kanon des Theophanes IV 3; Exapostilarion; Mittwoch (Orthros), Triodion des Joseph IX 3; Freitag (Orthros), Triodion des Joseph IX 3. 


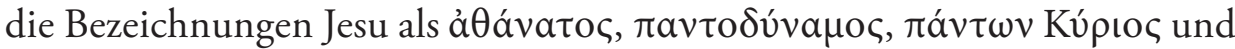

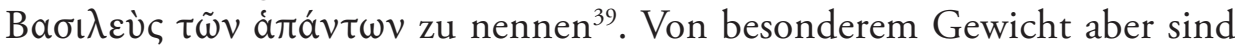
die beiden folgenden Prädikationen: Jesus wird zum einen als der „Schöpfer“ ${ }^{40}$ oder als der „Schöpfer aller“ bzw. der „Schöpfer des Alls“ ${ }^{41}$ bezeichnet, weil er den bereits verwesenden Lazarus in göttlicher Schöpfermacht durch sein Wort aus dem Tod ins Leben ruft. Und zum andern gilt Jesus als der „Spender des Lebens“" ${ }^{2}$, der in Person „die Auferstehung und das Leben“ ist $(\text { Joh } 11,25 b)^{43}$.

39 'A $\theta$ ávatoc: Kanon des Theophanes I 3. V 2; Freitag (Orthros), Triodion des Joseph IX

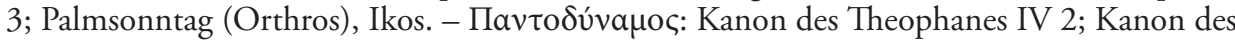
Kosmas IV 4; Stichira des Johannes 4; Freitag (Orthros), Triodion des Theodoros IX 3. -

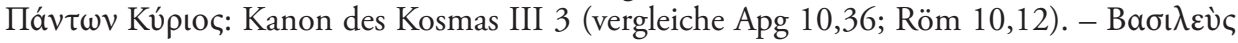

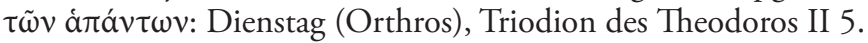

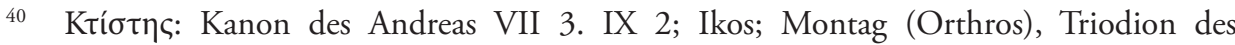
Theodoros VIII 3; Dienstag (Orthros), Triodion des Theodoros IX 3; Donnerstag (Orthros),

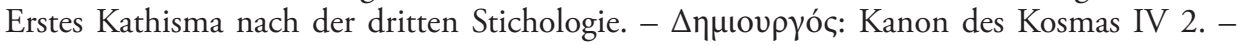

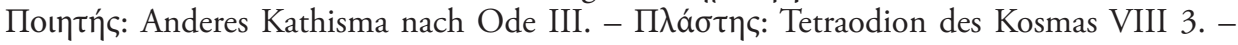

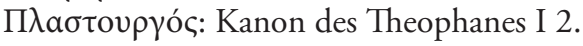

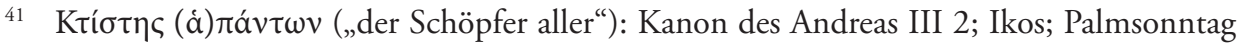

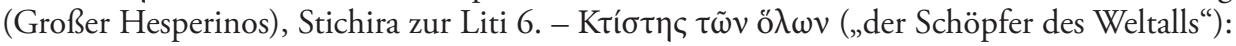

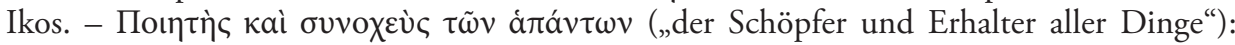

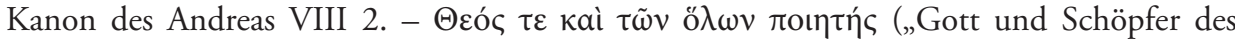
Weltalls"): Donnerstag (Hesperinos), Stichiron des Theodoros. - Tw̃v ő $\omega \omega \nu \pi \lambda a \sigma \tau o u \rho \gamma o ́ \varsigma$ („der Bildner des Weltalls“): Kanon des Andreas IX 8. Siehe ferner auch: Kathisma nach der

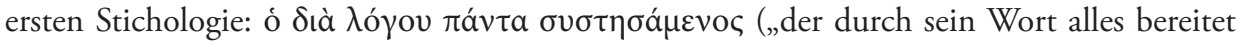

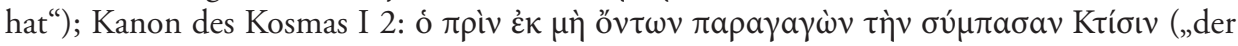
einst aus dem Nichts die ganze Schöpfung ins Dasein gerufen hat"). Ausdrücklich als

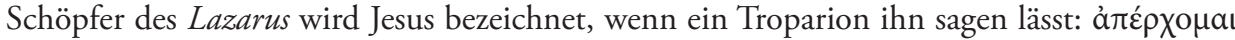

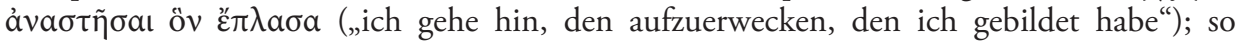
Mittwoch (Orthros), Erstes Kathisma nach der dritten Stichologie.

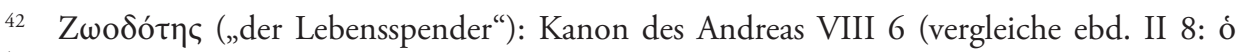
$\zeta \omega \omega \sigma \alpha \varsigma)$; Kathismata nach der ersten und zweiten Stichologie; Kanon des Theophanes I 5. III 3; Donnerstag (Orthros), Triodion des Theodoros IV 2. Vergleiche auch: Kanon des

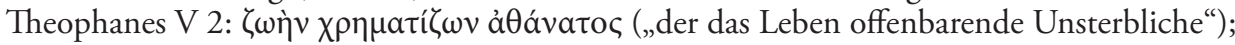

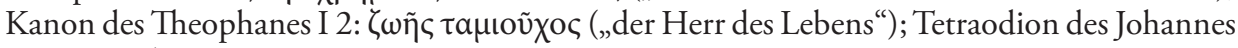

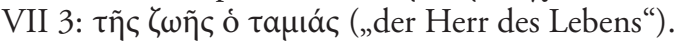

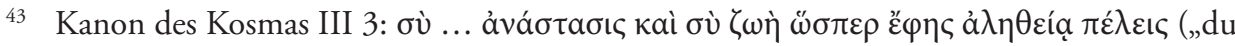
bist die Auferstehung, und du bist, wie du gesagt hast, in Wahrheit das Leben“); vergleiche Palmsonntag (Orthros), Kanon des Kosmas III 2. Siehe ferner etwa: Stichira des Johannes

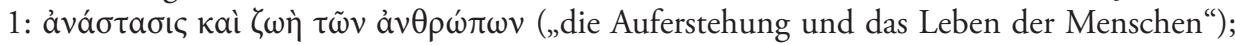

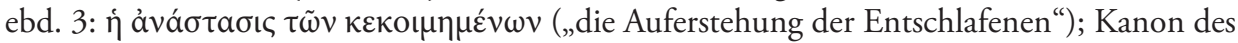

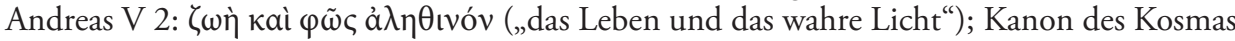

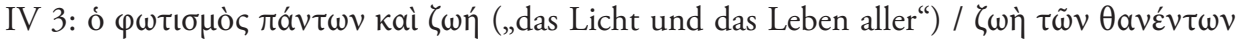
(„das Leben der Toten“); Kontakion (zitiert in Fußnote 85); Mittwoch (Orthros), Triodion

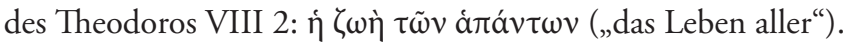


Wie Jesus in dem in Joh 11,1-44 berichteten Geschehen als wahrer Gott offenbar wird, so gibt er nach der Deutung der Hymnen hier zugleich auch seine wahre Menschheit zu erkennen. Obwohl er „als unbegrenzter Gott das All erfüllt", wird darin, dass er von einem Ort zum andern geht, sichtbar, dass er „ein sterblicher Mensch“ geworden ist ${ }^{44}$. Als Gott weiß er alle

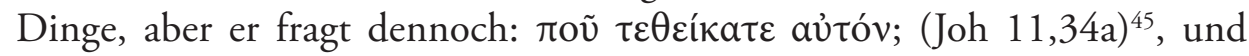
als man ihm die Stätte zeigt, weint er „nach dem Gesetz der menschlichen Natur“46 über den Tod des Lazarus (V. 35) ${ }^{47}$. Er, der „mit dem Vater gleichewig ist“ und „die Gebete aller annimmt“, betet am Grab des Lazarus „als Mensch" (Joh 11,41b.42) ${ }^{48}$ und erweist damit seinem himmlischen Vater die Ehre, wie sie ein Mensch ihm schuldig ist ${ }^{49}$. Wo immer die Hymnen die Motive erwähnen, die das Menschsein Jesu offenbaren, da stellen sie die Heilsbedeutung der Inkarnation heraus. Charakteristisch sind hier etwa die folgenden Wendungen, die uns jeweils in einem an Christus gerichte-

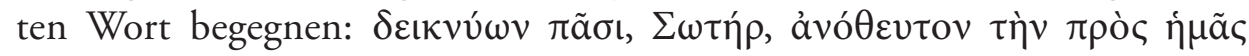

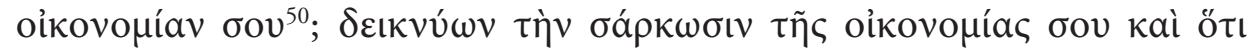

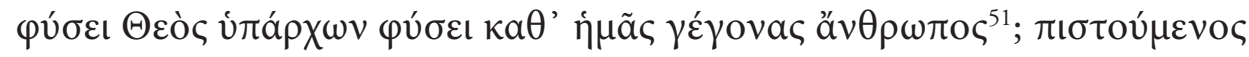

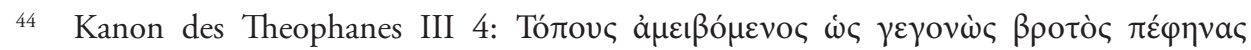

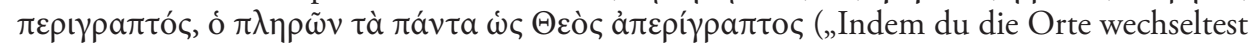
[d.h. von Ort zu Ort gingst], erschienst du als sterblich Gewordener begrenzt, du, der als unbegrenzter Gott das All erfüllt").

45 Kanon des Andreas I 6. III 2. IV 5. VI 2; Kanon des Kosmas I 3; Kanon des Theophanes III 3; Kathismata nach Ode III; Tetraodion des Kosmas VIII 3; Tetraodion des Johannes VIII 2; Stichira des Johannes 1.4. Vergleiche auch Stichira des Leon 1.

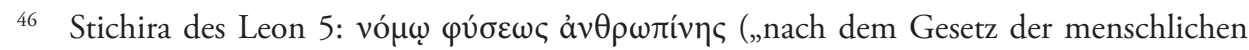

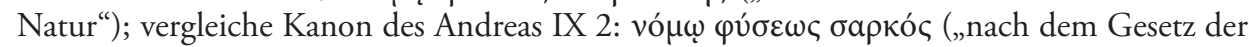
fleischlichen Natur").

47 Stichira des Leon 2.5; Kanon des Andreas I 4. II 5. III 5. IV 2.5. VI 2. VII 2. IX 2; Kanon des Kosmas III 2; Anderes Kathisma nach Ode III; Tetraodion des Johannes VI 2. IX 3; Tetraodion des Kosmas VII 2.

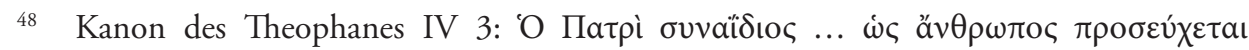

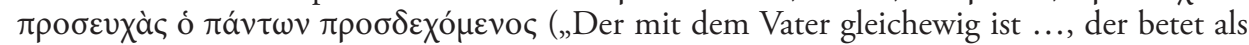
Mensch - er, der die Gebete aller annimmt"). Siehe auch ebd. 2: Jesus betet, obwohl er

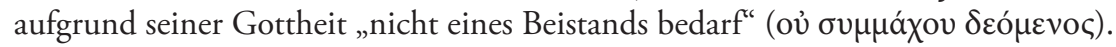

49 Kanon des Kosmas V 2; Tetraodion des Johannes IX 2. Zu Jesu Gebet als Ausdruck seiner wahren Menschheit siehe ferner: Anderes Kathisma nach Ode III; Tetraodion des Johannes VIII 4.

50 Kanon des Andreas I 6 („,allen, Heiland, deine uns geltende unverfälschte Heilsordnung

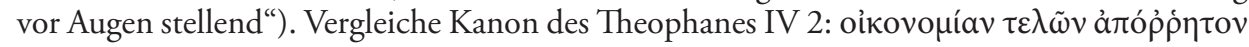
(„die unsagbare [oder: geheimnisvolle] Heilsordnung vollendend“).

51 Kanon des Andreas I 4 („die deiner Heilsordnung entsprechende Inkarnation vor Augen stellend - nämlich: dass du, obwohl du von Natur Gott bist, von Natur uns entsprechend [d.h. unserer Natur entsprechend] ein Mensch wurdest“). 


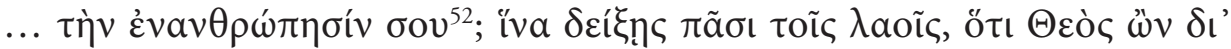

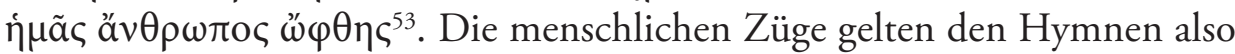

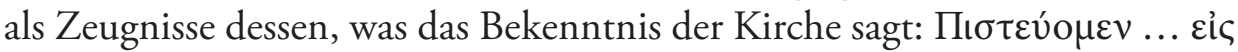

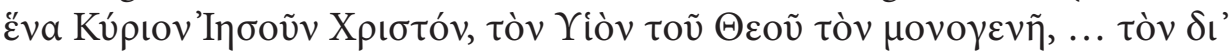

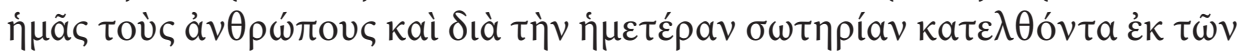

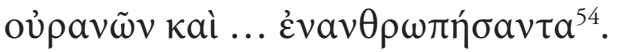

Man wird bezweifeln dürfen, dass in der Erzählung Joh 11,1-44 bereits die beiden „Naturen“ Jesu und das Verhältnis zwischen ihnen in der differenzierenden Weise im Blick sind, wie die Hymnen es darstellen. Gleichwohl bringen die Hymnen zutreffend zur Sprache, dass in der Auferweckung des Lazarus der handelt, den der Prolog des Johannesevangeliums als den präexistenten göttlichen Logos beschreibt (Joh 1,1-9) und von dem er sagt

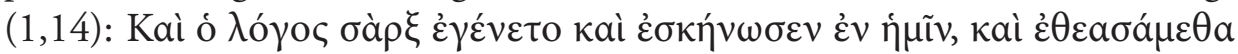

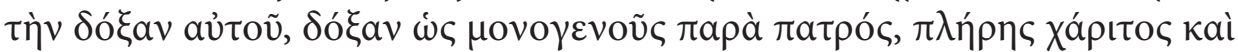
$a ̀ \lambda \eta \theta \varepsilon i ́ a \varsigma$. Dass in der Auferweckung des Lazarus die göttliche $\delta$ ó $\xi a$ offenbar wird, die dem menschgewordenen und in den Tod am Kreuz gehenden Gottessohn eignet, wird in Joh 11 ausdrücklich gesagt ${ }^{55}$. In den beiden - oben besonders hervorgehobenen - Prädikationen "Schöpfer" und „Spender des Lebens" sind deshalb zentrale Aussagen des Johannesevangeliums durchaus angemessen aufgenommen. Dass der Mensch Jesus von Nazareth der „Schöpfer" ist, ergibt sich aus Joh 1,3, wo es von dem, der dann $\sigma a ́ p \xi$ wird, heißt:

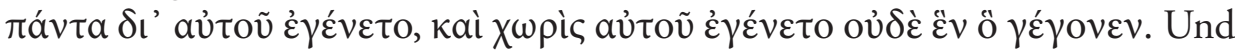
die Bezeichnung Jesu als „Spender des Lebens" hat ihr Fundament vor allem in dem solennen $\varepsilon \dot{\gamma} \dot{c} \varepsilon \dot{\jmath} \mu \mathrm{l}$ - Wort Joh 11,25b.c.26, das im Zentrum der Erzählung 11,1-44 steht ${ }^{56}$.

\section{IV}

Unsere bisherigen Überlegungen galten den erzählerischen und zugleich theologisch relevanten Motiven des Evangelienberichtes von der Auferweckung des Lazarus. Nunmehr ist die entscheidende theologische Aussage in den Blick zu fassen, um die es in dem Text Joh 11,1-44 geht.

\footnotetext{
52 Kanon des Andreas IV 5 („deine Menschwerdung gültig bezeugend“ [zur Übersetzung

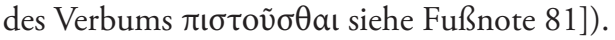

53 Kanon des Andreas VII 2 (,auf dass du allen Völkern vor Augen stelltest, dass du, obwohl du Gott bist, um unsertwillen als Mensch erschienen bist").

54 Symbolum Nicaeno-Constantinopolitanum.

55 Siehe die Verse 11,4 und 11,40.

56 Siehe außerdem: Joh 3,14-16.36; 4,14; 5,24.40; 6,35.47-51.54.63.68; 10,10b.28; 17,2; 20,31 .
} 
Wie bereits bemerkt wurde, handelt die Erzählung von der Auferweckung des Lazarus keineswegs nur von Lazarus selbst, sondern in ihr ist von einem jeden Menschen die Rede, zu dessen Rettung der Sohn Gottes den Tod erlitten hat. Was diesem Menschen widerfährt, wenn er zum Glauben an Christus als seinen Erlöser kommt, das wird in Joh 11,1-44 narrativ zum Ausdruck gebracht. Die Auferweckung des Lazarus will also nicht nur als eine Auferweckung aus dem physischen Tod verstanden sein. Sie steht vielmehr zugleich für die Auferweckung aus dem geistlichen Tod, dem jeder Mensch aufgrund seiner Sünde verfallen ist, und für das Zum-Glauben-Kommen, mit dem der Mensch das ewige Leben empfängt. Die Erzählung Joh 11,1-44 schildert mithin das Wunder der Befreiung aus dem Sündentod.

Die Lazarus-Hymnen bringen diesen Gedanken eindrucksvoll zur Sprache. Hier ist zunächst auf die sechste Ode des Kanon des Andreas von Kreta hinzuweisen. In den Strophen 1-7 redet der vom Tode auferweckte

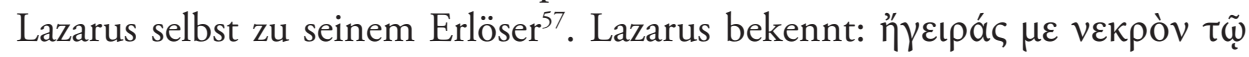
$\pi \rho o \sigma \tau a ́ \gamma \mu \alpha \tau i ́ ~ \sigma o v^{58}$ („du hast mich Toten auferweckt durch deinen Befehl“),

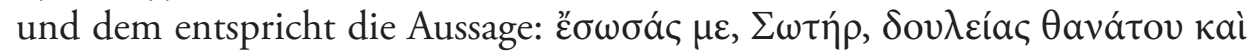

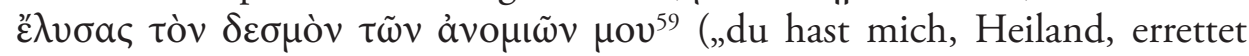
von der Knechtschaft des Todes und gelöst die Fessel meiner Sünden“). Die gottesdienstliche Gemeinde, die in die sechste Ode einstimmt, weiß sich mit Lazarus identisch und bekennt ihre eigene Befreiung von den Banden der Sünde ${ }^{60}$. In diesem Sinn ist auch das - ebenfalls in der „Ich“-Rede gehaltene - Troparion des Johannes gemeint:

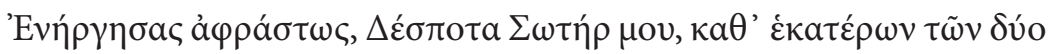

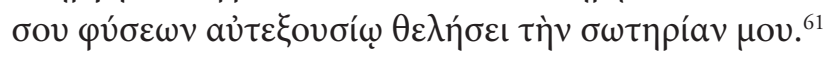

„Du hast auf unsagbare Weise, Herr, mein Heiland, kraft jeder deiner beiden Naturen in selbstmächtigem Willen meine Errettung bewirkt.“

Die zitierten „Ich“-Reden sind Äußerungen des durch Jesu schöpferisches Wort gewirkten Glaubens. Dass der vom Tode auferweckte Lazarus zum Glauben an seinen Retter kommt, ist vorausgesetzt, wenn es in den Hymnen

\footnotetext{
57 Dass es sich um Worte des Lazarus handelt, ergibt sich aus der dritten Strophe, wenn es

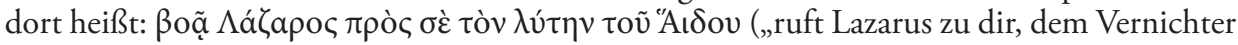
des Hades").

58 Kanon des Andreas VI 2-6.

59 Kanon des Andreas VI 1.

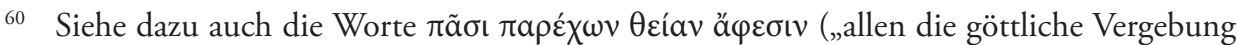
gewährend“) im Kontakion (zitiert in Fußnote 85) und im Ikos (zitiert in Fußnote 78).

${ }^{61}$ Tetraodion des Johannes IX 5.
} 
heißt, dass er anbetend vor Christus niederfällt ( $\pi \rho 0 \sigma \kappa v v \varepsilon \tilde{v})^{62}$, ihn preist

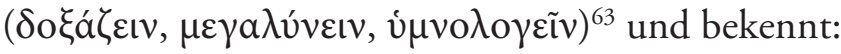

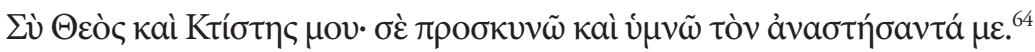

„Du bist mein Gott und Schöpfer. Dich bete ich an, und ich preise dich, der mich auferweckt hat."

Lazarus repräsentiert dabei zugleich diejenigen, die in diesen seinen Lobpreis einstimmen: die Jünger Jesu bzw. die zum Glauben kommenden Zeugen des Geschehens ${ }^{65}$ - und ebenso die Gemeinde, in deren Gottesdienst die Hymnen erklingen ${ }^{66}$. Wie einst die Kinder beim Einzug in Jerusalem ${ }^{67}$, so huldigt jetzt die Gemeinde dem, der sie vom Sündentod erlöst hat ${ }^{68}$.

Dass die durch Christus geschenkte Befreiung aus dem Sündentod von den Glaubenden immer neu in Anspruch genommen wird, bringen nicht wenige Hymnen zum Ausdruck ${ }^{69}$. Exemplarisch seien die drei folgenden Troparia zitiert:

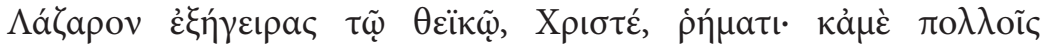

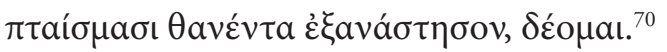

„Du hast Lazarus auferweckt, Christus, durch das göttliche Wort. Erwecke - ich bitte dich - auch mich auf, den an vielen Sünden Gestorbenen."

62 Kanon des Andreas IV 4; Kathisma nach Ode III.

63 Kanon des Andreas II 3; Dienstag (Orthros), Triodion des Theodoros IX 3; Freitag (Orthros), Triodion des Theodoros VIII 3.

64 Kanon des Andreas VII 3. Vergleiche ferner: Stichira des Leon 4.5; Tetraodion des Kosmas VII 2.3; Tetraodion des Johannes VII 3.

65 Stichira des Leon 2; Tetraodion des Kosmas IX 2; Exapostilarion; Mittwoch (Hesperinos), Stichiron des Theodoros.

66 Tetraodion des Kosmas IX 3; Anderes Exapostilarion; Donnerstag (Orthros), Erstes Kathisma nach der dritten Stichologie; Donnerstag (Hesperinos), Stichiron des Theodoros.

${ }^{67}$ Exemplarisch: Palmsonntag (Orthros), Erstes Kathisma nach der zweiten Stichologie.

68 Exemplarisch: Palmsonntag (Orthros), Kathismata nach der ersten Stichologie.

69 Siehe außer den im Folgenden zitierten Texten: Kanon des Kosmas V 3; Tetraodion des Johannes VI 4; Stichira des Johannes 8; Montag (Orthros), Erstes Kathisma nach der zweiten Stichologie; Montag (Orthros), Triodion des Joseph IX 2; Dienstag (Hesperinos), Stichira des Joseph 1; Mittwoch (Orthros), Erstes Kathisma nach der zweiten Stichologie; Mittwoch (Orthros), Triodion des Joseph III 3. VIII 2. IX 3; Mittwoch (Hesperinos), Stichira des Joseph 2; Donnerstag (Orthros), Erstes Kathisma nach der zweiten Stichologie; Donnerstag

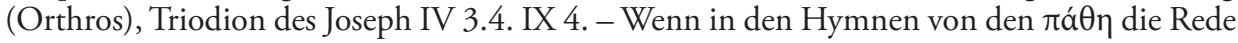
ist, dann sind die „Sündenleidenschaften“ gemeint.

70 Kanon des Theophanes III 5. 


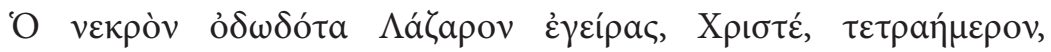

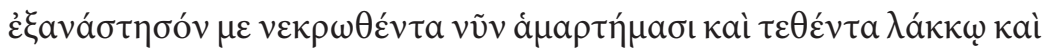

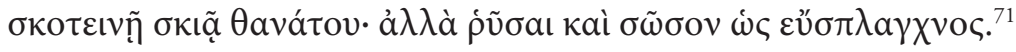

„Der du den toten und schon verwesenden Lazarus auferweckt hast, Christus, - ihn, der bereits vier Tage im Grabe lag: Erwecke mich auf, der jetzt in Sünden gestorben und der Grube und dem finsteren Schatten des Todes übergeben ist. Ja, erlöse und errette mich als der, der barmherzig ist."

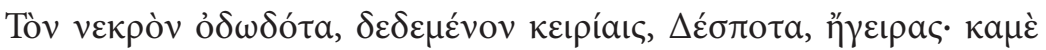

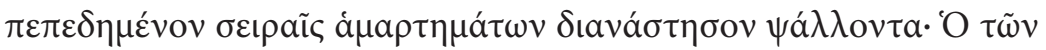

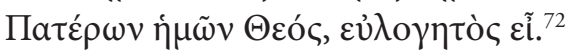

„Den schon verwesenden Toten, der von Grabtüchern umwunden war, hast du, Herr, auferweckt. Auch mich, der umgarnt ist von den Stricken der Sünden, richte auf, damit ich singe: Gott unserer Väter, gepriesen bist du!“

\section{V}

Zuletzt sind noch zwei weitere theologische Aussagen der Hymnen zu bedenken, die in der Sache aufs Engste miteinander verbunden sind.

1. Die Hymnen setzen die Auferweckung des Lazarus betont zu der Auferstehung Jesu in Beziehung, wobei diese in ihrem unlöslichen Zusammenhang mit dem Tod Jesu am Kreuz gesehen ist ${ }^{73}$. In der Auferweckung des

71 Kanon des Theophanes V 4.

72 Tetraodion des Johannes VII 4.

73 Es entspricht diesem Tatbestand, dass im Orthros des Lazarussamstags das sonntägliche

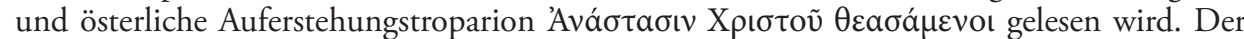

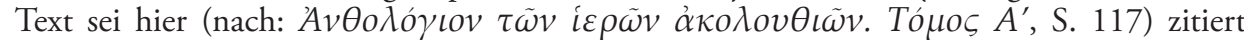

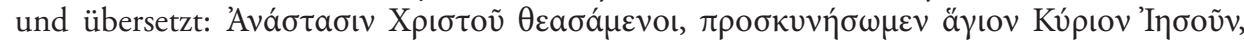

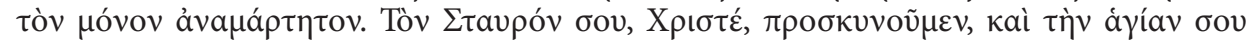

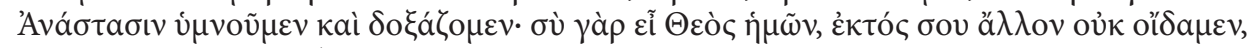

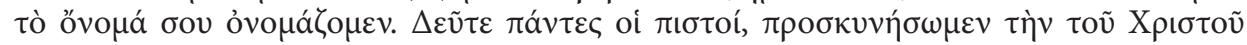

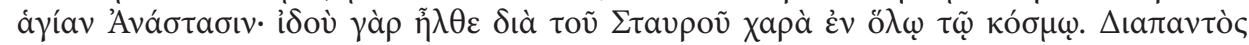

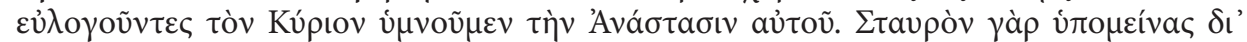

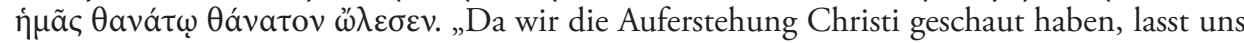
anbeten den heiligen Herrn Jesus, der allein ohne Sünde ist. Vor deinem Kreuz, o Christus, fallen wir nieder, und wir besingen und verherrlichen deine heilige Auferstehung; denn du bist unser Gott, außer dir kennen wir keinen anderen, deinen Namen rufen wir an. Kommt, alle ihr Gläubigen, lasst uns die heilige Auferstehung Christi anbeten; denn siehe: durch das Kreuz ist Freude in die ganze Welt gekommen. Allezeit loben wir den Herrn und besingen wir seine Auferstehung. Denn er hat um unsertwillen das Kreuz erduldet und durch den (= seinen) Tod den Tod vernichtet." 
Lazarus - so das Zeugnis der Hymnen - erweist sich Jesus als der, der den $\theta$ ávatoc bzw. den "Aı $\delta \eta \varsigma^{74}$ bezwingt und seiner Herrschaft ein Ende setzt ${ }^{75}$. Die Dichter können gelegentlich so reden, als sei der Sieg über den Tod bereits in der Auferweckung des Lazarus errungen worden ${ }^{76}$. Solche Formulierungen wollen jedoch im Kontext der Lazarus-Hymnen insgesamt verstanden sein, für die - dem neutestamentlichen Zeugnis entsprechend - die Erkenntnis grundlegend ist, dass die Überwindung des Todes in Jesu Tod und Auferstehung geschehen ist. Der „Sieger über den Tod“ bzw. der „Vernichter des Hades"77 ist Jesus also als der gekreuzigte und auferstandene Herr. Was er als der Gekreuzigte und Auferstandene ist, das bestimmt ihn aber in seiner ganzen

74 Das Wort "Aı $\delta\rceil \varsigma$ wird in den Hymnen vielfach personifiziert verwendet und stellt deshalb

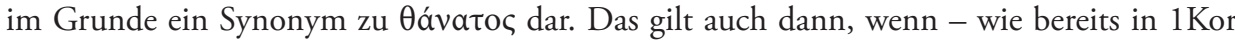
15,55 v.l.; bei Meliton von Sardes, Passa-Homilie 102, und in den in Fußnote 3 erwähnten

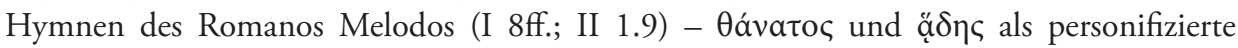
Größen nebeneinander erscheinen (zum traditionsgeschichtlichen Hintergrund siehe Hiob 38,17 LXX; Jes 22,15 LXX; Offb 1,18; 6,8; 20,13f.). Die Unterscheidung ist in diesem Fall

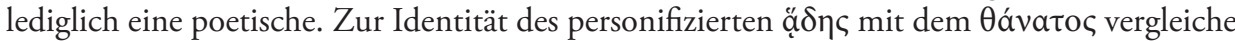
auch die Belege aus den Schriften der Kirchenväter bei Geoffrey W.H. Lampe, A Patristic Greek Lexicon, Oxford $1961={ }^{5} 1978$, S. 32a s.v. å̊j门ৎ D.

75 Siehe etwa Stichira des Leon 2.3; Kanon des Andreas I 7. II 8. III 6. IV 6. VI 3. IX 6; Kanon des Theophanes I 3. IV 4; Kanon des Kosmas III 4. IV 4. V 3; Tetraodion des Johannes VII 3; Exapostilarion; Anderes Exapostilarion; Stichira des Johannes 2; Montag (Hesperinos), Stichiron des Theodoros; Dienstag (Orthros), Triodion des Theodoros IX 3; Dienstag (Hesperinos), Stichiron des Theodoros; Mittwoch (Hesperinos), Stichira des Joseph 1; Donnerstag (Orthros), Erstes Kathisma nach der dritten Stichologie; Donnerstag (Orthros), Triodion des Joseph VIII 3; Donnerstag (Orthros), Triodion des Theodoros IV 3. VIII 3. IX 3; Donnerstag (Hesperinos), Stichira des Joseph 2; Donnerstag (Hesperinos), Stichiron des Theodoros; Freitag (Orthros), Erstes Kathisma nach der dritten Stichologie.

76 Als Beispiel kann der erste Satz des Ikos dienen, der im Orthros des Palmsonntags rezitiert

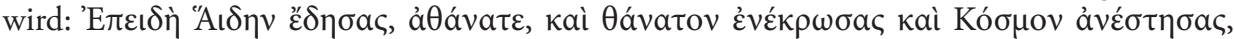

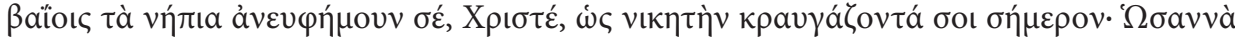

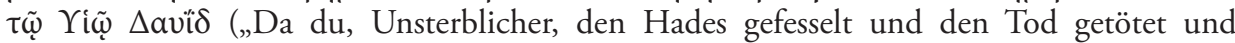
die Menschheit auferweckt hast, haben die Unmündigen mit Zweigen dich, Christus, unermüdlich als Sieger gepriesen, und sie rufen dir heute laut zu: Hosianna dem Sohne

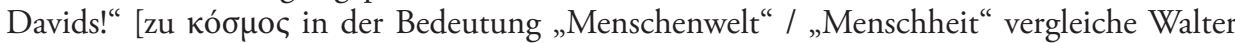
Bauer, Kurt und Barbara Aland, Griechisch-deutsches Wörterbuch zu den Schriften des Neuen Testaments und der frühchristlichen Literatur, Berlin ${ }^{6} 1988$, Sp. 907 s.v. 6]). Siehe ferner etwa: Stichira des Leon 3; Kanon des Andreas I 7. IV 6; Kanon des Theophanes I 3. IV 4; Kanon des Kosmas V 3; Donnerstag (Orthros), Triodion des Joseph VIII 3; Donnerstag (Hesperinos),

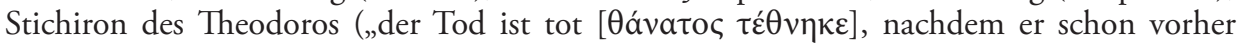
gemerkt hat, das Lazarus die Toten verlässt").

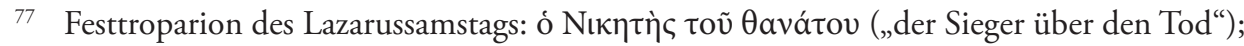

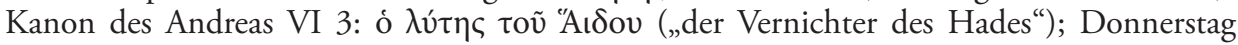

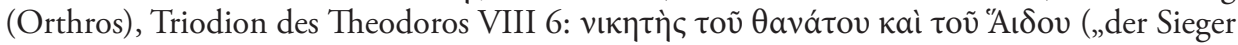
über den Tod und den Hades"). 
Existenz, und daraus folgt, dass er die Auferweckung des Lazarus eben als der wirkt, der zur Überwindung des Todes am Kreuz sterben und am dritten Tage von den Toten auferstehen wird. Das Wunder, das Lazarus widerfährt, kann deshalb zum einen als die „Präfiguration“ des Kreuzesgeschehens ${ }^{78}$ und als Hinweis auf die Bedeutung der Passion und des Kreuzestodes Jesu ${ }^{79}$ bezeichnet werden; und zum andern wird von diesem Wunder gesagt, dass Jesus mit ihm seine Auferstehung und den in ihr errungenen Sieg „im Voraus gültig bezeugt " ${ }^{\text {" }}$. Der Gedanke, dass Jesus durch das Lazarus-Wunder im Voraus seine Auferstehung "gültig bezeugt " ${ }^{\text {"81, }}$, spielt in den Hymnen eine hervorgehobene

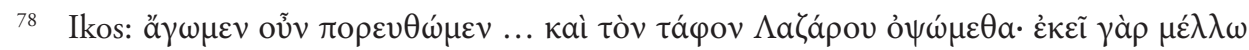

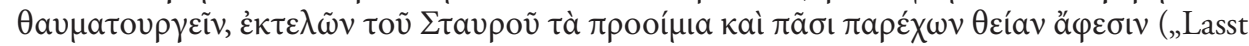
uns nun hingehen ... und das Grab des Lazarus schauen; denn dort will ich ein Wunder vollbringen, um die Präfiguration des Kreuzes[geschehens] ins Werk zu setzen und allen die göttliche Vergebung zu gewähren“). Zu dem zitierten und übersetzten Text ist sprachlich zweierlei anzumerken: 1. Das präsentische Partizipium conjunctum wird bereits im Neuen Testament als einem Finalsatz gleichwertig verwendet; siehe Friedrich Blaß, Albert Debrunner, Friedrich Rehkopf, Grammatik des neutestamentlichen Griechisch, Göttingen ${ }^{17} 1990, \$ 418,4$. Dieser Gebrauch des Partizipiums liegt m. E. in unserem Troparion vor - und ebenso auch in den Troparia, die in Fußnote 82, in Fußnote 84 und bei Fußnote 90 zitiert werden. 2.

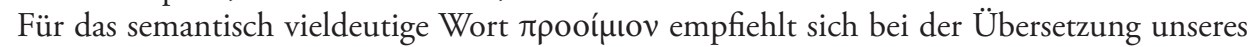
Troparions die Wiedergabe mit dem Fremdwort „Präfiguration“ (vergleiche dazu auch Stichira des Leon 3, zitiert bei Fußnote 94). Der Ikos kennzeichnet die Auferweckung des Lazarus also als einen vorweg gegebenen Hinweis auf das, was am Kreuz geschehen und durch Christi Kreuzestod gewirkt werden wird.

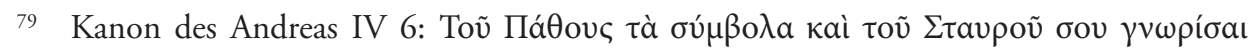

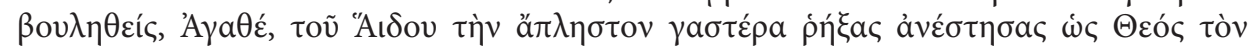

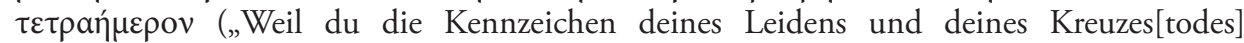
offenbaren wolltest, o Guter, hast du den unersättlichen Magen des Hades zerschmettert und

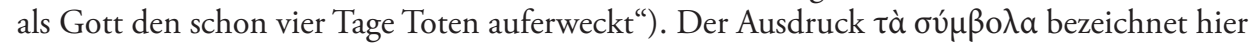
eine Größe, an der etwas zuverlässig erkannt werden kann (vergleiche zu dieser Bedeutung: Stichira des Johannes 4; Donnerstag (Orthros), Triodion des Joseph IV 5). Gemeint ist also: An der Auferweckung des Lazarus zeigt sich zuverlässig, was in Jesu Passion und Kreuzestod

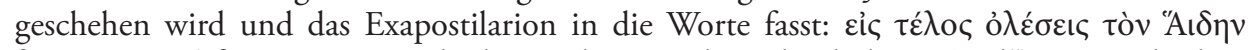

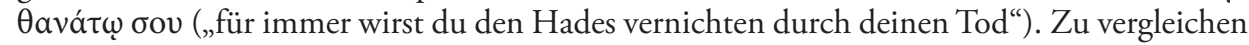
sind ferner auch die Aussagen über das Kreuz bzw. die Kreuzigung Jesu in: Kanon des Andreas I 7; Dienstag (Hesperinos), Stichira des Joseph 1; Mittwoch (Orthros), Triodion des Joseph VIII 4; Donnerstag (Hesperinos), Stichira des Joseph 2; Palmsonntag (Orthros), Kanon des Kosmas I 2.

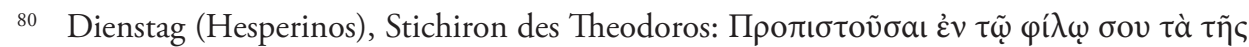

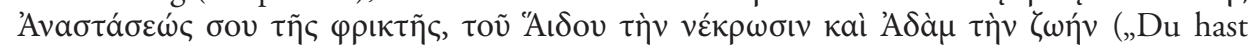
an deinem Freund im Voraus gültig bezeugt, was in deiner furchterregenden Auferstehung geschehen ist: des Hades Tod und Adams [Auferweckung zum] Leben"). Zu dem Verbum $\pi \rho о \pi \iota \sigma \tau o \tilde{\sigma} \theta$ aı siehe die nächste Fußnote.

81 „Gültig bezeugen“ oder „unter Beweis stellen“ ist m. E. der Sinn des in den Hymnen

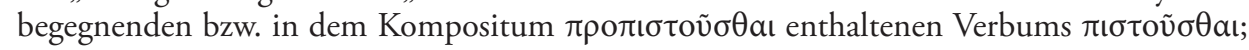




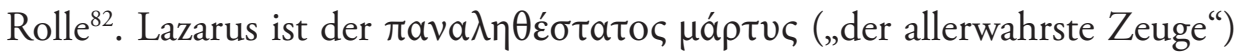
der Auferstehung Jesu ${ }^{83}$, weil Jesus an ihm eben jene Macht über den Tod unter Beweis stellt, die in seiner eigenen Auferstehung offenbar wird ${ }^{84}$. Indem

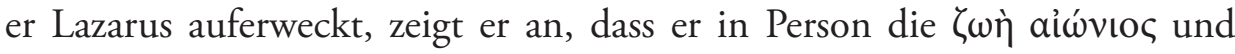
deshalb „das Leben aller" ist ${ }^{85}$. Von daher ergibt sich dann eine wichtige Konsequenz für das Verständnis der Auferweckung des Lazarus. Sie bedeutet nach dem Zeugnis der Hymnen entschieden mehr, als dass der Tote in das irdische Leben zurückgerufen wird, und sie gilt ihnen auch keineswegs nur als die bloße Verheißung und Verbürgung einer künftigen Auferweckung zum ewigen Leben. Mit seiner Auferweckung empfängt Lazarus vielmehr bereits die $\zeta \omega \grave{\eta}$

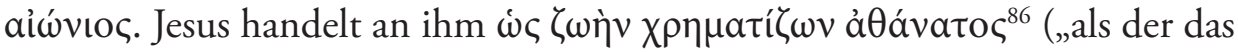
Leben offenbarende Unsterbliche") - und das heißt: Er gewährt ihm eben jene Gabe, die in gleicher Weise den durch Lazarus repräsentierten Glauben-

siehe dazu auch die Zitate in Fußnote 32 (Tetraodion des Johannes VI 2) und in Fußnote 52 (Kanon des Andreas IV 5).

82 Siehe vor allem: Kanon des Andreas IX 3 (zitiert in Fußnote 84); ferner: Stichira des Leon 4; Kanon des Kosmas I 4. IV 2; Tetraodion des Kosmas IX 3. Vergleiche auch Palmsonntag

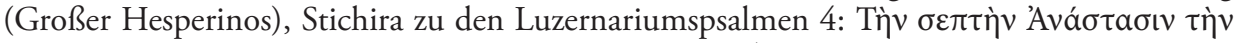

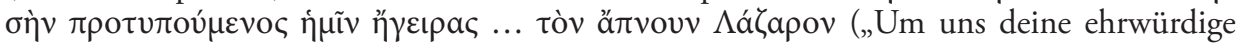
Auferstehung im Voraus zu offenbaren [s.o. Fußnote 78], hast du den toten Lazarus auferweckt").

83 Tetraodion des Johannes IX 3.

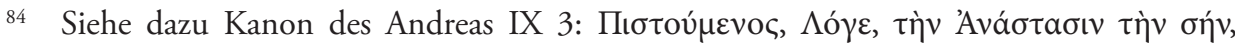

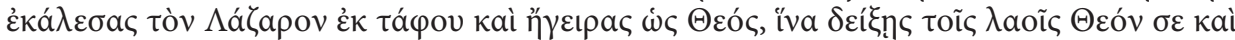

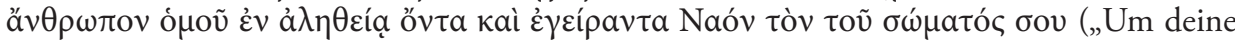
Auferstehung, o Wort, gültig zu bezeugen [s.o. Fußnote 78], hast du Lazarus aus dem Grab gerufen und als Gott ihn auferweckt, damit du den Völkern zeigtest, dass du in Wahrheit zugleich Gott und Mensch bist und so auch den Tempel deines Leibes auferweckt hast"). Das Troparion verweist keineswegs zufällig auf den höchst gewichtigen Text Joh 2,19.21, dem die nicht weniger gewichtige Aussage von Joh 10,17f. an die Seite zu stellen ist.

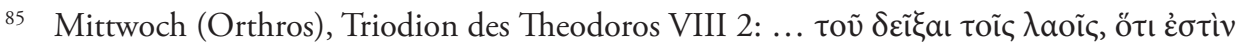

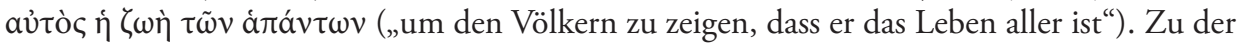
hier vorliegenden Bezugnahme auf Joh 11,25b siehe auch die in Fußnote 43 angeführten

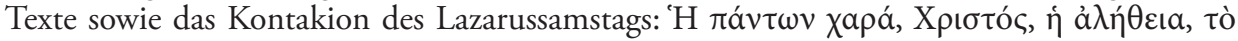

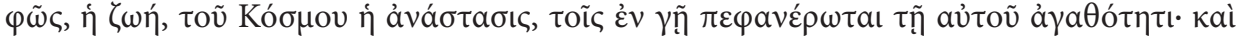

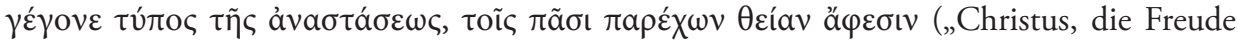
aller, die Wahrheit, das Licht, das Leben, die Auferstehung der Menschheit, ist denen, die auf Erden sind, in seiner Güte erschienen; und er wurde zum Urbild der Auferstehung, da

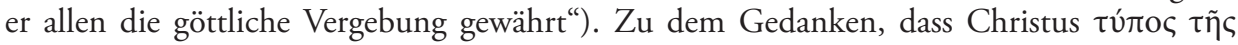

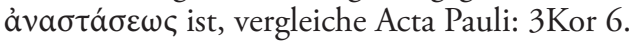

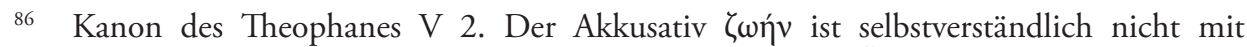

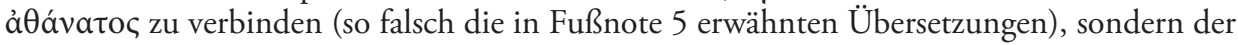
Satz besagt: Jesus, der selbst unsterblich ist, „offenbart“ in der Auferweckung des Lazarus das ewige Leben. 
den zuteil wird: die Unsterblichkeit ${ }^{87}$. Auch wenn der Begriff der ả $\theta a v a \sigma i ́ a$ im Vierten Evangelium nicht erscheint, so bringen die Lazarus-Hymnen mit ihm doch sehr wohl zur Sprache, was an zentralen Stellen des Evangeliums über die den Glaubenden mit ihrer Auferweckung aus dem geistlichen Tod

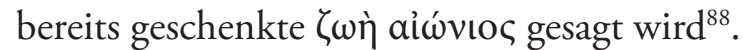

2. Damit stehen wir bereits bei der Beziehung zwischen der Auferweckung des Lazarus und der eschatologischen Auferstehung der Toten. Von dieser Beziehung spricht das Festtroparion des Lazarussamstags, das mit den Worten beginnt:

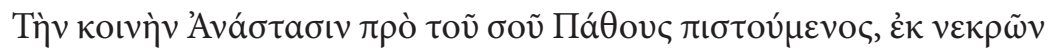

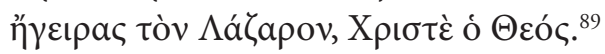

„Um die allgemeine Auferstehung vor deinem Leiden gültig zu bezeugen $^{90}$, hast du Lazarus von den Toten auferweckt, Christus, Gott.“

Wird in diesem Satz gesagt, dass Christus mit der Auferweckung des Lazarus die allgemeine Totenauferstehung "gültig bezeugt" habe ${ }^{91}$, so heißt es in anderen Texten, dass er sie hier ,im Voraus angekündigt / kundgegeben “92 oder „im Voraus angezeigt "993 hat. Fragen wir, wie das gemeint ist, so gibt uns das im Großen Hesperinos des Palmsonntags gesungene sechste Stichiron zur

87 Mittwoch (Orthros), Triodion des Joseph VIII 4 (zitiert bei Fußnote 17); Freitag (Orthros), Triodion des Joseph IX 3 (zitiert bei Fußnote 17); Palmsonntag (Großer Hesperinos), Anderes

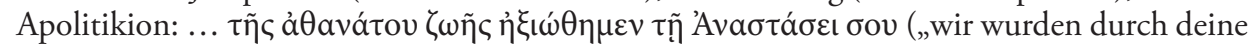
Auferstehung des unsterblichen Lebens gewürdigt").

88 Siehe dazu neben Joh 11,25f. vor allem Joh 3,14-16.36a; 5,24; 6,40.47.54; 10,10.28; 20,21.

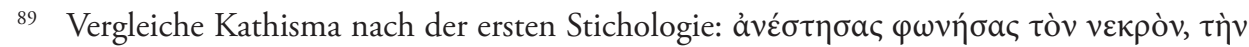

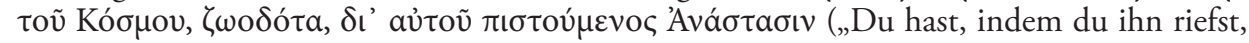
den Toten auferweckt und so, Lebensspender, durch ihn die Auferstehung der Menschheit gültig bezeugt").

90 Zum finalen Gebrauch des präsentischen Partizipium conjunctum s.o. Fußnote 78.

${ }_{91} \mathrm{Zu} \pi \iota \sigma \tau o \tilde{\sigma} \sigma$ aı siehe Fußnote. 81 .

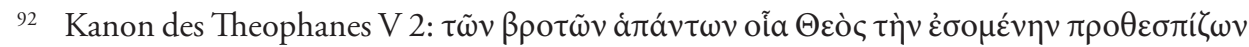

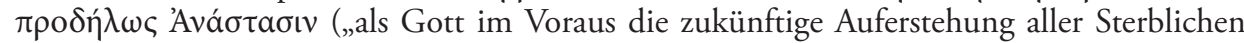

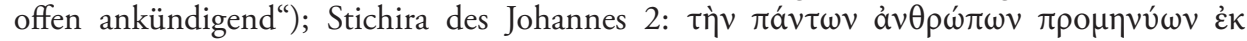

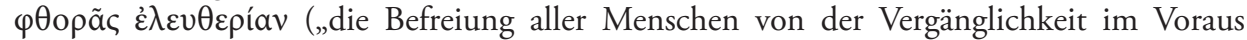

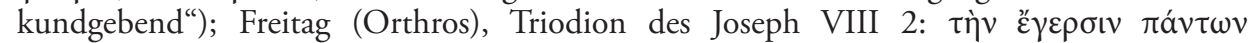
$\pi \rho \eta_{\eta} v \dot{\omega} \omega v$ („die Auferweckung aller im Voraus kundgebend“); Palmsonntag (Großer

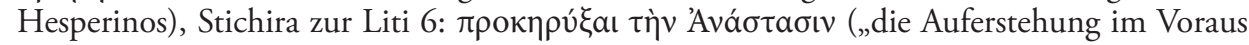
kundzutun").

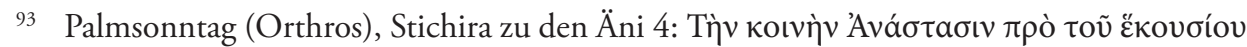

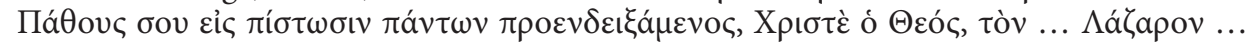

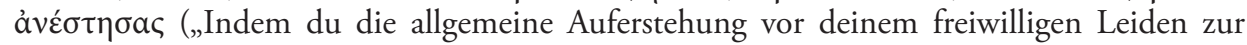
Beglaubigung für alle im Voraus anzeigtest, Christus, Gott, hast du Lazarus auferweckt“). 
Liti einen wichtigen Fingerzeig. Nach diesem Stichiron besteht die „Vorherverkündigung" der Totenauferstehung darin, dass sich an Lazarus das Wort

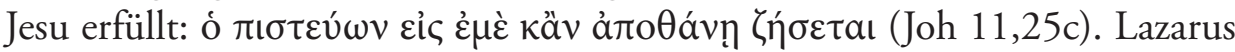

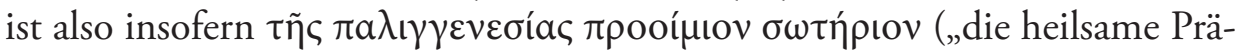
figuration der Wiedergeburt [d.h. der den Menschen neumachenden Auferstehung]") geworden ${ }^{94}$, als an ihm sichtbar wird, dass dem durch Jesus aus dem Sündentod auferweckten und an ihn glaubenden Menschen die zukünftige Auferstehung bereits gültig zugeeignet ist und dass sie auch durch den Tod nicht mehr in Frage gestellt werden kann. Wir können somit zusammenfassend formulieren: Die Auferweckung des Lazarus ist nach dem Zeugnis der Hymnen ein Vorschein des Ostersieges Christi, in dem die Auferstehung der an Christus Glaubenden definitiv entschieden und begründet ist - und zwar als eine Wirklichkeit, die sie schon jetzt in ihrem Sein zeichnet und bestimmt.

\section{Terminologischer Anhang}

Den griechischen Begriffen wird, wo es angezeigt ist, in Klammern die erasmische Aussprache beigefügt.

Äni: Die Aĩvor (Ainoi) sind die zum Morgengottesdienst (Orthros) gehörenden Laudes-Psalmen Ps 148-150. An Sonn- und Festtagen werden an verschiedenen Stellen Stichira ( $\rightarrow$ Stichiron) zu den Psalmversen hinzugefügt.

Apolitikion: 'Aло入vтíkıv (Apolytikion) heißt das einem bestimmten Werktag, Sonntag oder Festtag zugeordnete $\rightarrow$ Troparion, weil es unter anderem am Ende des Abendgottesdienstes (Hesperinos) vor der Entlassung

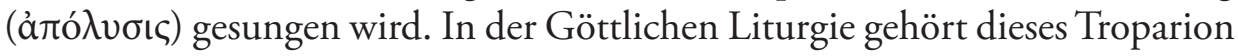
zu den Hymnen, die auf den Kleinen Einzug folgen.

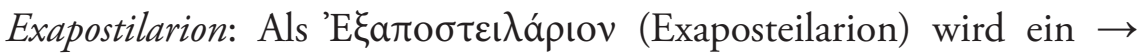
Troparion bezeichnet, das im Morgengottesdienst (Orthros) auf den $\rightarrow \mathrm{Ka}$ non folgt und den $\rightarrow$ Äni unmittelbar voraufgeht.

Ikos: Der Oĩkoৎ (Oikos) ist ein $\rightarrow$ Troparion, das ebenso wie das $\rightarrow$ Kontakion ursprünglich Bestandteil einer umfangreichen frühbyzantinischen Kirchendichtung war, die im Gottesdienst gesungen, später jedoch durch den jüngeren $\rightarrow$ Kanon verdrängt wurde. Der Ikos folgt im Morgengottesdienst (Orthros) zusammen mit dem ihm voraufgehenden Kontakion auf die sechste $\rightarrow$ Ode des Kanons.

\footnotetext{
94 Stichira des Leon 3. Zu $\pi \alpha \lambda \iota \gamma \gamma \varepsilon v \varepsilon \sigma i ́ a$ als Bezeichnung für die eschatologische Auferstehung

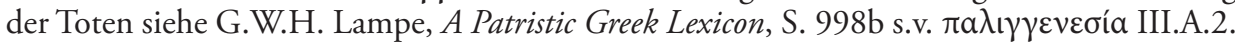
Für $\pi$ pooíftov gilt das in Fußnote 78 Gesagte.
} 
Irmos: Eipuóc (Heirmos) heißt ein als Leitstrophe dienendes $\rightarrow$ Troparion, das hinsichtlich der Silbenzahl, der Betonung und der Melodie das Modell für die auf es folgenden Troparia liefert. Im $\rightarrow$ Kanon ist der Irmos jeweils das erste Troparion einer jeden $\rightarrow$ Ode. An Sonn- und Festtagen wird der Irmos häufig am Ende einer Ode wiederholt.

Kanon: Der Kavẃv ist die letzte Hochform der byzantinischen Kirchendichtung und bildet insbesondere ein wesentliches Element des Morgengottesdienstes (Orthros). Ein voll entwickelter Kanon besteht aus neun bzw. - da die zweite $\rightarrow$ Ode ausschließlich an bestimmten Tagen der Großen Fastenzeit gesungen wird - aus acht Oden. In der Großen Fastenzeit weist der Kanon vielfach eine auf drei und gelegentlich auch eine auf vier Oden reduzierte Gestalt auf. Ein nur drei Oden umfassender Kanon heißt $\rightarrow$ Triodion, ein nur vier Oden umfassender Kanon $\rightarrow$ Tetraodion.

Kathisma: Hinsichtlich des Begriffs KáӨı $\sigma \mu \alpha$ ist zwischen dem Psalmen-Kathisma und dem poetischen Kathisma zu unterscheiden. Als Psalmen-Kathisma wird jeweils eine der zwanzig Psalmengruppen bezeichnet, in die der alttestamentliche Psalter für die fortlaufende Lesung im Gottesdienst unterteilt ist (vgl. $\rightarrow$ Stichologie). In der Regel sind für den Morgengottesdienst (Orthros) zwei oder drei Kathismata und für den Abendgottesdienst (Hesperinos) ein Kathisma vorgesehen. Bei den poetischen Kathismata handelt es sich um Troparia ( $\rightarrow$ Troparion), bei deren Gesang man sitzen darf. Im Morgengottesdienst gibt es poetische Kathismata, die auf die Lesung eines Psalmen-Kathisma folgen, wie auch solche, die sich an die dritte $\rightarrow$ Ode des $\rightarrow$ Kanons anschließen.

Kontakion: Das Kovtákıov ist ein $\rightarrow$ Troparion, das ebenso wie der $\rightarrow$ Ikos ursprünglich Bestandteil einer umfangreichen frühbyzantinischen Kirchendichtung war. Diese wurde im Gottesdienst gesungen, später jedoch durch den jüngeren $\rightarrow$ Kanon verdrängt. Das Kontakion folgt im Morgengottesdienst (Orthros) auf die sechste $\rightarrow$ Ode des Kanons. In der Göttlichen Liturgie gehört es zu den Hymnen, die im Anschluss an den Kleinen Einzug gesungen werden.

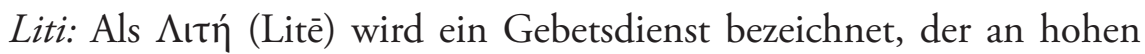
Festtagen unmittelbar mit dem Abendgottesdienst (Hesperinos) verbunden ist und zu dem eine im Kirchenraum vollzogene Prozession der Liturgen gehört. Während der Prozession werden Troparia ( $\rightarrow$ Troparion) gesungen.

Luzernariumspsalmen: Als Luzernariums- oder Lichterpsalmen gelten vier miteinander verbundene Psalmen, die zu den ältesten Elementen des 
Abendgottesdienstes (Hesperinos) gehören: Ps 140, Ps 141, Ps 129 und Ps 116 (Septuaginta-Zählung; Hebräische Bibel: Ps 141, Ps 142, Ps 130 und Ps 117). Zum Gesang dieser Psalmen, zu denen Stichira ( $\rightarrow$ Stichiron) hinzugefügt werden, werden im Kirchenraum alle Lichter angezündet.

Ode: Die poetische ' $\Omega\left\llcorner\delta \eta^{\prime}\right.$ ist ein zu einem $\rightarrow$ Kanon gehörender Hymnus. Eine Ode besteht aus drei bis acht metrisch-musikalisch gleichgestalteten Troparia $(\rightarrow$ Troparion), wobei das erste Troparion den $\rightarrow$ Irmos, d.h. die Leitstrophe bildet.

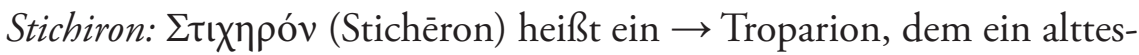
tamentlicher Psalmvers $(=\sigma \tau i ́ \chi 0 \varsigma)$ voraufgeht. Stichira werden unter anderem mit den $\rightarrow$ Äni und mit den $\rightarrow$ Luzernariumspsalmen verbunden.

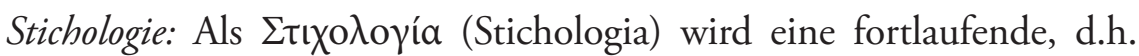
Vers für Vers erfolgende Lesung aus dem Psalter bezeichnet (vgl. $\rightarrow$ Kathisma).

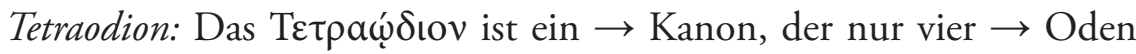
umfasst. Tetraodia werden ausschließlich in der Großen Fastenzeit gesungen, und zwar im Morgengottesdienst (Orthros) an den Samstagen der zweiten bis sechsten Fastenwoche (in der sechsten Woche ist dies der Lazarussamstag) sowie am Karsamstag.

Triodion: Das Tpıọ́dıv ist ein $\rightarrow$ Kanon, der nur drei $\rightarrow$ Oden umfasst. Triodia haben ihren Ort in der Großen Fastenzeit. Sie werden vor allem - außer am Dienstag und Donnerstag der Karwoche - von Montag bis Freitag im Morgengottesdienst (Orthros) gesungen. Von daher trägt auch das liturgische Buch, das die Eigentexte für die Vorfastenzeit und die Große Fastenzeit einschließlich der Karwoche enthält, den Namen „Triodion“.

Troparion: Der Begriff Tpoтápıov bezeichnet eine hymnische Einheit, die mit der Strophe eines Kirchenliedes der westlichen Tradition vergleichbar ist. Viele Hymnen bestehen aus mehreren Troparia. Das für einen bestimmten Wochentag, Sonntag oder Festtag vorgesehene Troparion wird $\rightarrow$ Apolitikion genannt. 